\title{
Pneumatic Manipulator Control System Based on PLC
}

\author{
Linghao Shen*
}

Zhejiang Ocean University, Zhoushan 316022, China. E-mail: 865077503@qq.com

Abstract: The main purpose of this manipulator is to transport small cargoes. The traditional hand claw is used to grasp goods. PLC control system is mainly used for operation. At the same time, PLC is used to realize the automatic/manual control of the manipulator.

Keywords: Manipulator; PLC Control; Programmable Logic Controller

In fact, the industrial manipulator is an extension of the function of human arms, replacing human beings to complete monotonous and heavy work in high-decibel, air-polluted workshops, and to a certain extent, reducing the cost while greatly improving the production efficiency. The manipulator can carry the target object, load and unload numerical control machine tools, to achieve industrial production automation, so the automatic production line equipped with manipulator is applied to not only reduce labor costs but also promote efficient production of manipulator basic structure: the actuator is manipulator for various activities of the entity. The layout of actuator will have a great influence on the working performance of manipulator, There are many basic forms of which the main four types are: articulated manipulator, cylindrical coordinate manipulator, cylindrical-coordinate manipulator and spherical coordinate manipulator $^{[1]}$. For example, in terms of the five-axis paint manipulator, its structure is similar to human arm, its elbow jointing to achieve a number of degrees of freedom and suitable for working in the range of motion is smaller and suitable for operating near the body of the transmission form, by the big arm, forearm, waist and wrist composition, and it has a relatively broad prospect $^{[2]}$.

\section{Introduction to pneumatic tech- nology}

Pneumatic drive transmits energy and signals through compressed air. It is a common driving mode with hydraulic drive, which promotes the piston motion through air pump compression. The higher the pressure, the greater the flow rate, and the faster the movement speed.

Pneumatic components play an important role in electronic mechanical integration and electronic components integration.

Pneumatic systems are usually composed of actuators, control components, power components and various auxiliary components.

Advantages: simple structure, high working efficiency, low energy loss.

Generally, air is used as a working medium where the environmental pollution is low.

This is an open-access article distributed under the terms of the Creative Commons Attribution Non-Commercial License (http://creativecommons.org/licenses/by-nc/4.0/), which permits unrestricted non-commercial use, distribution, and reproduction in any medium, provided the original work is properly cited. 
The start-up is fast, with little lag and high precision.

Disadvantages: use compressible gas, with poor stability, insufficient working pressure, insufficient compensation function.

\section{Design of pneumatic loop}

Pneumatic cylinder claw grabs by the five-way valve and control valve, and solenoid valve has no electricity, no gas in the air pump. After electrifying, slide valve controls air current from the left into the pneumatic cylinder, the piston moves down, the rotary gear is driven by rack, and gripper begins clamping. When air flows into the opposite direction, the piston moves up, and the hand claw loosens ${ }^{[3]}$.

At the same time, the speed regulating valve can change the grasping speed of hand by changing the speed of air flow.

Pneumatic arm is lifted by each of the three-way valve and control valve, electricity and gas flow into the entrance to push the piston upward, driving the guide bar upward and making manipulator rise. When the airflow flows in from another entry, the piston and the push rod move in opposite directions. The speed of manipulator, by the same token, can be changed by the change of gas flow velocity ${ }^{[4]}$.

\subsection{Telescopic boom cylinder}

Composed of the three-way valves and speed regulating valves, after the power, the gas flows in after the piston outward motion, driving the telescopic boom outward extension. In terms of the manipulator, when the gas flows in from another direction, the piston moves in the opposite direction, the telescopic boom shortens inward, and the manipulator retracts. Similarly, changing the flow rate of gas can change the velocity of contraction.

\subsection{Claw}

Using connecting rod type gripper, the gas flowing into the speed regulating valve pushes the piston through the end rack and the middle rack, driving the meshing gear to rotate, and making the gripper open or closed. The gripper's opening range is determined by grasping the work piece.

\section{Motion process of control loop}

Press the start button to obtain the telescopic cylinder horizontal elongationand manipulator from the originand stop the elongation cylinder whose elongation extends downwards.At the same time, the hand grab, electromagnetic valve, power cylinder piston move down, and the rack gear rotates. PAWS grab, grab the artifacts-telescopic cylinder returns, up to the limit position, he stopped rising-motor drive gear, restores manipulator, reaches the right limit, stops turning motion-telescopic cylinder elongation down, stretches to the limit position, stops the elongation and electromagnetic valve power, gripper and loosen the telescopic cylinder return, up to the limit position. He stopped rising-motor drive gear, making the manipulator reverse rotation, to the left limit, stoping rotary movement, retracting the telescopic cylinder horizontally and moving the manipulator to the origin position.

\section{PLC control principle}

This manipulator is a cylindrical coordinate pneumatic manipulator. The robotic arm has three degrees of freedom, namely horizontal expansion, and contraction. A rise or fall in the vertical direction; left and right rotation of the base. The claw part of the manipulator can also complete the function of catching and putting the work piece ${ }^{[5]}$. Rotary motion uses pneumatic motor drive, and the rest of the action uses pneumatic cylinder drive, namely three or two five-way solenoid valve, which controls three pneumatic cylinders and motors, so that the manipulator can complete telescopic, lifting, rotation and take action.

This paper uses modular design program, and manipulator control program is divided into automatic program, manual program, and public program.

When running, the utility program will be executed first. If manual program is selected, I2.0 will be switched on to manual program; if automatic program is selected, I2.4 will be switched on to automatic program.

\subsection{I/O port allocation}

\subsubsection{Manual control mode}

Motion of manipulator controlled by I0.1-I0.4, sets about turning simultaneously. The rise and fall, to prevent action between the chain instead of running at the 
same time. Limit switch I0.2 normally open contacts and control of the rotating Q0.3 and Q0.4 coil in series, the rotation of the manipulator must rise to the position, prevent collision with other machine. The manipulator rotates the end to allow telescopic cranes and lifting arms to work.

\subsubsection{Automatic control mode}

When working automatically, it switches on the normally closed contact of single-step switch I2.2, switches on the conversion permit sign, and allows the conversion between steps.

At the same time, the continuous switch I 2.4 is on, and the system is in the original state. When the manipulator falls to the lower limit switch I0.1, "Clamping" step I2.1 is on, clamping valve Q0.1 is placed, the work piece is clamped, the manipulator rises, and so on. The manipulator will go down step by step.

\subsection{Hardware selection}

Choose Siemens S7-200 PLC, which is widely used in China. The specific model is SIMATIC S7-200 CPU224. The machine integrates 14 input /10 output with 24 digital quantity I/O points. 7 extension modules can be connected, up to 168 channels digital quantity I/O points or 35 channels analog quantity I/O points. $16 \mathrm{~K}$ byte program and data storage space. 6 independent $30 \mathrm{kHz}$ high speed counters, 2 independent $20 \mathrm{kHz}$ high speed pulse output, with PID controller. 1 RS485 communication/programming port with PPI communication protocol, MPI communication protocol and free mode communication capability. The I/O terminal row can be easily disassembled. It is a controller with strong control ability $^{[6]}$.

\subsection{Buffer device}

The cylinder end buffer device was used this time. The principle is that when the piston moves some distance from the cylinder head, the end cover and the piston can form a buffer chamber. The buffer chamber can generate the back-pressure resistance which can be used to slow down and stop the piston, to avoid collision ${ }^{[7-9]}$.

For heavy load low speed pneumatic manipulator, the maximum acceleration is generally taken below 5 $\mathrm{m} / \mathrm{s}^{2}$, and for light load high speed pneumatic manipulator, the maximum acceleration is generally taken $5 \sim 10$ $\mathrm{m} / \mathrm{s}^{2}$.
During the horizontal exercise:

$$
F=P_{s} A-F_{i}
$$

The residual speed $V_{\mathrm{r}}$

$$
V_{\mathrm{r}}=\frac{V_{\mathrm{o}}}{\sqrt{1-\frac{\alpha_{\max }}{\mathrm{F}}}}
$$

\section{Conclusion}

Based on the relevant principles of PLC technology and combined with the structure of the manipulator, this paper designs a PLC-based pneumatic manipulator control system which is also based on the PLC control mode, pneumatic loop mode, and program structure ${ }^{[10]}$. Theoretically, the manual and automatic multiple angle motion manipulator control is realized, which further improves the control accuracy of the manipulator and makes the system more practical. At the same time, the buffer device is added to improve the safety of operation. With the help of computer technology and electronic science technology, the PLC-based control system has strong anti-interference ability and can further develop the "pneumatic-PLC-sensor" automatic control system. If it forms an independent system with the relay control system, the reliability is higher and the probability of operation errors is lower.

In terms of economic benefits, the rapid handling of small goods is realized, labor is liberated, and operating costs are reduced to a certain extent.

\section{References}

1. Chen X, Luo M, Zhao J, et al. The design of the control system based on PLC large stroke and heavy load manipulator (in Chinese). Automation \& Instrumentation 2013; (3): 60-62.

2. Li J. Based on PLC control system design of manipulator. Coal Mine Machinery 2012; 33(10): 147148.

3. Zhang Y. Research on the harvesting robot system for the measurement of 3D motion [master's thesis]. Zhenjiang: Jiangsu University; 2008.

4. Yang W. Design and analysis of apple harvesting robot's manipulator [master's thesis]. Zhenjiang: Jiangsu University; 2009.

5. Chen Y, Zou X, Xu D, et al. Mechanism design and kinematics simulation of litchi picking manipulator. Journal of Machine Design 2010; 27(5): 31-34. 
6. Jiang H. Design of motion control system of industrial manipulator based on PLC (in Chinese) [master's thesis]. Nanjing: Nanjing University of Information Science \& Technology; 2012.

7. Huang J. Simulation research of POWERCUBE modular manipulator in MSRS and ODE environment (in Chinese) [master's thesis]. Dalian: Dalian Jiaotong University; 2010.
8. Lu X, Yang X. Manipulator-Theory and application (in Chinese). Beijing: China Railway Publishing House Co., Ltd; 1985. p. 33-56.

9. Zhang J. Industrial robot (in Chinese). Beijing: Beijing Institute of Technology Press; 1994. p. 66-91.

10. Chen Z. Programmable controller (PLC) system design (in Chinese). Beijing: Publishing House of Electronic Industry; 2007. 